\title{
Pprl: The Key Protein in Response to DNA Damage in Deinococcus
}

\author{
Huizhi Lu and Yuejin Hua* \\ MOE Key Laboratory of Biosystems Homeostasis and Protection, Institute of Biophysics, College of Life Sciences, Zhejiang \\ University, Hangzhou, China
}

OPEN ACCESS

Edited by:

Brian C. Schaefer,

Uniformed Services University of the Health Sciences, United States

Reviewed by:

Lydia Contreras,

University of Texas at Austin,

United States

Elena K. Gaidamakova, Henry M Jackson Foundation for the Advancement of Military

Medicine (HJF), United States

Sangyong Lim,

Korea Atomic Energy Research

Institute (KAERI), South Korea

*Correspondence:

Yuejin Hua

yjhua@zju.edu.cn

Specialty section:

This article was submitted to

Cell Death and Survival,

a section of the journal

Frontiers in Cell and Developmental

Biology

Received: 22 October 2020

Accepted: 17 December 2020

Published: 18 January 2021

Citation:

Lu H and Hua Y (2021) Pprl:

The Key Protein in Response to DNA

Damage in Deinococcus.

Front. Cell Dev. Biol. 8:609714.

doi: 10.3389/fcell.2020.609714
Deoxyribonucleic acid (DNA) damage response (DDR) pathways are essential for maintaining the integrity of the genome when destabilized by various damaging events, such as ionizing radiation, ultraviolet light, chemical or oxidative stress, and DNA replication errors. The Pprl-DdrO system is a newly identified pathway responsible for the DNA damage response in Deinococcus, in which Pprl (also called IrrE) acts as a crucial component mediating the extreme resistance of these bacteria. This review describes studies about Pprl sequence conservation, regulatory function, structural characteristics, biochemical activity, and hypothetical activation mechanisms as well as potential applications.

Keywords: Pprl, DNA damage response, switch, Pprl-DdrO system, Deinococcus

\section{INTRODUCTION}

Deoxyribonucleic acid (DNA) damage occurs when the genome is exposed to exogenous and endogenous hazards, leading to imperfection and instability of the genetic information (Pilzecker et al., 2019). If not repaired in a timely and accurate manner, accumulating mutations will result in severe effects, such as cancer, and even lead to cell death. To cope with DNA damage, organisms have evolved various DNA damage repair pathways, including nucleotide excision repair, nonhomologous end joining, homologous recombination, mismatch repair, and base excision repair (Boulton et al., 2002).

The SOS response involves a common mechanism that is induced after DNA damage occurs in various bacteria (Radman, 1975; Butala et al., 2008, 2011). In the SOS response system, LexA functions as a transcriptional repressor that mediates the transcription of $r e c A$ and other SOS genes. When sensing DNA damage, RecA forms filaments with ssDNA in the presence of ATP, causing the autocleavage of LexA. The decrease in the cellular pool of LexA leads to dissociation of SOS boxbound LexA, thus initiating SOS gene transcription. The upregulation of SOS genes is repressed by abundant LexA after the damage is repaired (Butala et al., 2008, 2011; Figure 1).

As one of the most radio-resistant organisms on Earth, bacteria belonging to the genus Deinococcus can withstand a series of environmental stresses, such as high doses of ionizing radiation, UV radiation, oxidation, mitomycin $\mathrm{C}$, and long periods of desiccation, due to their extraordinary antioxidant system and DNA repair capability (Cox and Battista, 2005; Makarova et al., 2007; Blasius et al., 2008; Slade and Radman, 2011; Lim et al., 2019; Qi et al., 2020). Indeed, members of Deinococcus produce several antioxidants, including catalase, peroxidase, superoxide dismutase, carotenoids, and manganese ion antioxidant complex, to deal with oxidative stresses (Slade and Radman, 2011; Sharma et al., 2017). Deinococcus seldom invoke translesion synthesis and non-homologous end joining, but rather adopt homologous recombination to guarantee the fidelity of DNA repair (Slade and Radman, 2011). Several proteins, such as PprI, PprA, DrRRA, and 
OxyR, have been identified involving in the DNA damage response: PprI is the switch mediating the transcription of DDR genes, PprA contributes to UV radiation resistance and interacts with both DraTopoIB and the Gyrase A subunit, DrRRA cooperates with PprI and functions in gamma radiation resistance, and OxyR senses the presence of reactive oxygen species to regulate the antioxidant system (Chen et al., 2008; Wang et al., 2008, 2012, 2015; Bauermeister et al., 2009; Selvam et al., 2013; Kota et al., 2014).

However, unlike in most bacteria, the two encoded LexA in Deinococcus radiodurans do not participate in the induction of $\operatorname{rec} A$, although the autocleavage activity remains unchanged, indicating the malfunction of the classic SOS response system (Narumi et al., 2001; Sheng et al., 2004; Jolivet et al., 2006). Instead of the SOS system, a novel pathway has been found to be responsible for the DNA damage response in Deinococcus: two conserved proteins, $\mathrm{DdrO}$ - the repressor and PprI-the derepressor, comprise this unique response system (Devigne et al., 2015; Wang et al., 2015; Figure 1). To activate DDR genes participating in pathways such as DNA replication and stress response, PprI cleaves DdrO to deprive its DNA-binding ability after sensing DNA damage signals by unclear activation mechanisms (Wang et al., 2015).

This article summarizes the research progress on PprI in the last few years, mainly covering its structure and function. Potential applications and probable activation mechanisms of PprI in response to DNA damage as well as other oxidative stresses are also discussed.

\section{Pprl STRUCTURE REVEALS THREE DISTINCT DOMAINS}

As reported before, pprI from D. deserti shares 73 and $64 \%$ sequence identity with $D$. geothermalis and $D$. radiodurans homologs and can complement the loss of radiation resistance of pprI deletion in D. radiodurans (Vujicic-Zagar et al., 2009). It is also demonstrated that PprI from either D. geothermalis or $D$. radiodurans can cleave $\mathrm{DdrO}$ from either D. geothermalis or $D$. radiodurans, further demonstrating that PprI cleavage of DdrO as well as the PprI-DdrO response system is conserved among Deinococcus species (Lu et al., 2019).

The crystal structure of PprI from $D$. deserti was solved by Vujicic-Zagar et al. (2009), revealing that the protein consists of three domains: one zinc peptidase-like domain, one helixturn-helix motif, and one GAF-like domain. The N-terminal domain of PprI exhibits a zinc metallopeptidase fold and contains a conserved HEXXH sequence (Vujicic-Zagar et al., 2009). Ludanyi et al. (2014) and Wang et al. (2015) later proved that PprI functions as a protease targeting DdrO. Subsequent research on $\mathrm{HEXXH}$-related residues has indicated that $\mathrm{H} 82, \mathrm{E} 83, \mathrm{H} 86$, and E113 are indispensable for metal ion binding as well as the PprI protease function (Wang et al., 2015).

The middle region of PprI comprises an HTH domain that is usually responsible for DNA binding. Although some researchers doubt the DNA-binding ability of PprI based on the structural domain arrangement and alignment with ParB-DNA structure,
Lu verified the promoter-binding ability of PprI in vitro and in vivo (Vujicic-Zagar et al., 2009; Lu et al., 2012). He also proved that DNA binding is important for PprI function in response to DNA damage, and truncation of the $\mathrm{HTH}$ domain leads to loss of DNA affinity in PprI and the failure of RecA induction after radiation, as well as a decrease in stress resistance in D. radiodurans (Lu et al., 2012).

The C-terminus of PprI forms a GAF-like domain, which is one of the most widespread small molecule-binding domains responsible for binding allosteric regulatory molecules, named after a series of proteins consisting of GAF domains: cGMPspecific phosphodiesterases, adenylyl cyclases, and FhlA (Aravind and Ponting, 1997). Structural alignments revealed that the C-terminus is similar to the GAF domain in the Thermotoga maritima transcription factor IclR, Klebsiella pneumoniae CitA28, and Escherichia coli PDE2A that always participate in the binding of regulatory molecules such as cAMP and cGMP for the stress response. The comparison indicated that the C-terminus may be responsible for signal transduction, although this possibility needs further verification (Martinez et al., 2002; Sevvana et al., 2008).

\section{Pprl IS A GENERAL SWITCH REGULATING DDR GENES}

Seventeen years ago, both Earl and Hua found that the pprI (also called irrE) gene can regulate the expression of $\operatorname{rec} A$ gene and that its deletion would lead to the sensitivity of $D$. radiodurans to radiation (Earl et al., 2002; Hua et al., 2003; Gao et al., 2005). The disruption of pprI led to the decrease in resistance to gamma radiation, $\mathrm{UV}$ radiation, $\mathrm{H}_{2} \mathrm{O}_{2}$, and mitomycin $\mathrm{C}$ and left $\mathrm{DdrO}$ un-cleaved after radiation, while similar phenotypes were also detected in D. deserti (Vujicic-Zagar et al., 2009; Lu et al., 2012; Ludanyi et al., 2014; Wang et al., 2015).

To uncover the function role and regulation pathways of pprI in Deinococcus, proteomics and transcriptomics studies have been conducted. Proteomics research has revealed that various proteins are significantly upregulated by PprI after exposure to a low dose of gamma radiation. These proteins are involved in several different pathways, including DNA replication and repair, stress response, energy metabolism, transcriptional regulation, signal transduction, protein turnover, and chaperone functions (Lu et al., 2009). Later, microarrays and time-course sampling were applied to analyze the dynamic transcription of the pprI mutant strains compared with wild type. A total of 210 genes were found to be significantly induced in irradiated wild-type $D$. radiodurans but not in the irradiated p prI mutant strains. Consistent with the proteomics data, these genes participate in various pathways, indicating that $p p r I$ is a global regulator ( $\mathrm{Lu}$ et al., 2012). Part of these genes are regulated by PprI directly, such as $\operatorname{ppr} A, s s B$, and $r e c A$, whose promoter contains RDRM (radiation/desiccation response motif) site and repressed by DdrO (Liu et al., 2003; Makarova et al., 2007). Genes like DR0997 $(d d r I)$ and DR1114 (hsp20) that are also regulated by PprI without the conserved RDRM site are classified as indirectly regulated by PprI (Makarova et al., 2007; Lu et al., 2009; Singh et al., 2014; 


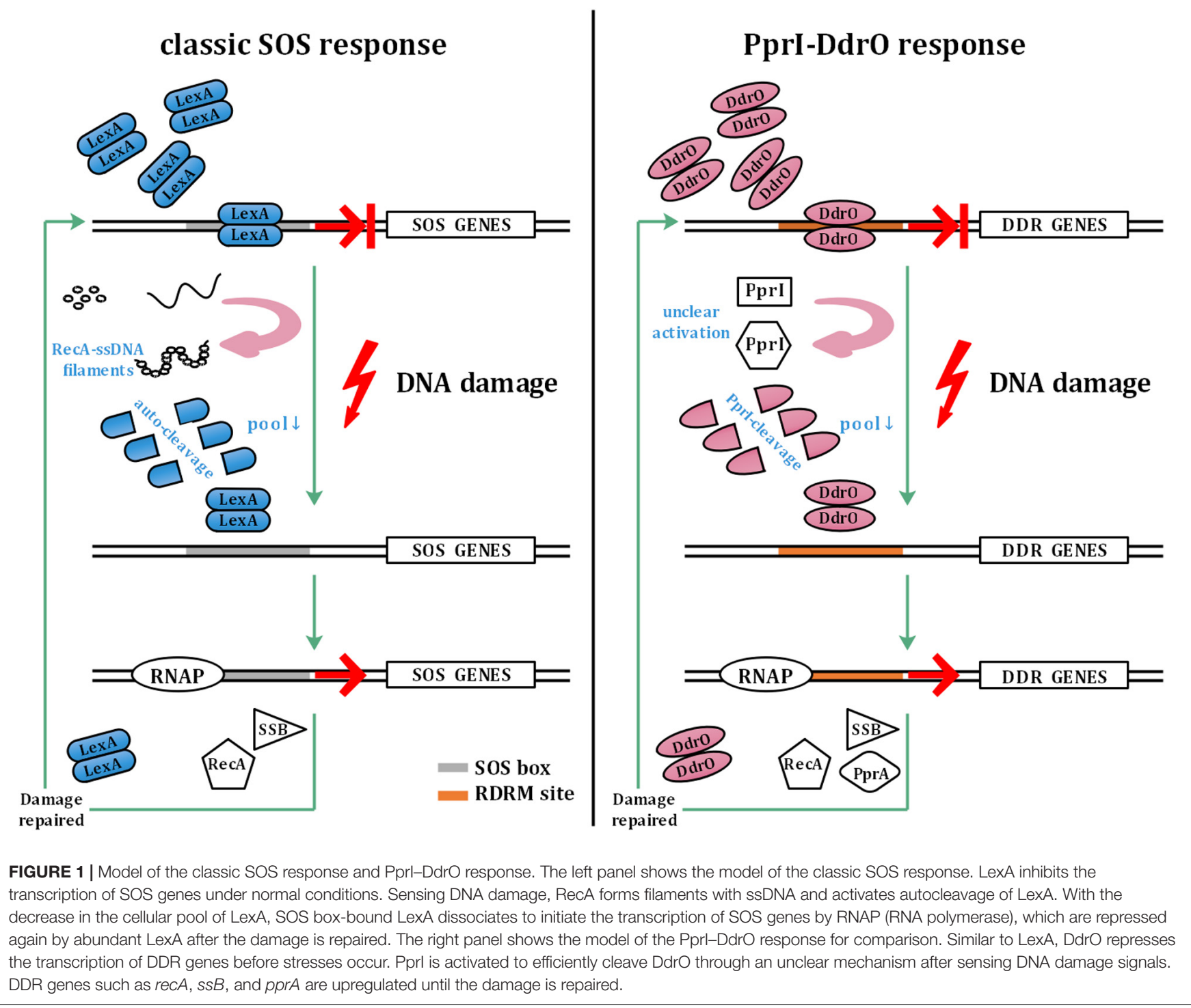

Yang et al., 2016). Moreover, Wang reported that DrRRA and PprI may collaborate to defend against environmental stresses (Wang et al., 2008, 2012).

\section{REPRESSION AND DEREPRESSION MECHANISMS OF THE PprI-DdrO SYSTEM}

It is reported that proteins of COG2856, such as YdcM, tend to fuse with XRE (xenobiotic-response element) family proteins to form operons regulating cascade downstream. PprI is also belonging to COG2856, indicating the cooperation with an XRE family protein similar to other proteins in toxinantitoxin systems (TAS) (Bose et al., 2008; Makarova et al., 2009). The mechanism by which PprI regulates a series of DDR genes is revealed along with the discovery of its action on the transcription repressor DdrO (Ludanyi et al., 2014;
Wang et al., 2015). DdrO, a component in this DNA damage response system, belonging to the XRE family, is a transcriptional repressor that forms dimers and specifically binds to the promoter region of DDR genes, including $d d r O$ itself, to repress DDR gene transcription under normal conditions (de Groot et al., 2019; Lu et al., 2019). These promoter regions contain a conserved 17-bp palindromic motif named RDRM (Makarova et al., 2007). After sensing DNA damage, DdrO is cleaved by PprI, which in turn relieves the transcriptional repression of DNA damage response genes. Thus, the repressor DdrO, in coordination with the protease PprI, constitutes the novel pathway mediating the DNA damage response in Deinococcus.

The detail of how the repressor DdrO works in the system remained unclear until the structure of DdrO was determined. The crystal structure of DdrO from D. geothermalis was solved by Lu et al. (2019). The results showed that DdrO is composed of eight $\alpha$-helices, containing an $\mathrm{HTH}$-containing $\mathrm{N}$-terminal domain and a novel fold of C-terminal domain. Although the 
structure of DdrO and promoter DNA in complex is not yet available, comparison of DdrO with other XRE family protein complexes and biochemical studies have revealed a conserved binding mode and recognition/binding residues in the $\mathrm{HTH}$ motif. It is verified in the article that the solvent-exposed residues such as R22, R28, K30, Y42, and D45 in DG-DdrO are essential for binding affinity. As for the RDRM sequence recognition and binding, both variation of the conserved base pairs and length shortening impaired the binding of DG-DdrO. In conclusion, the extended dimeric interaction in DdrO is essential for binding to RDRM-containing sequences (Makarova et al., 2007; Lu et al., 2019; Chen et al., 2020). Besides, apart from this NTD dimerization, Arjan de Groot also revealed a CTD dimerization of DdrO that is quite different to the already known XRE family proteins such as SinR (de Groot et al., 2019).

Analysis of the novel fold in the DdrO C-terminus exhibits enrichment of hydrophobic residues forming a stable hydrophobic core. The cleavage destabilizes the C-terminal hydrophobic core and disrupts the DdrO dimer, terminating the transcriptional repression of DDR genes as the specific DNA affinity of DdrO requires its dimeric conformation ( $\mathrm{Lu}$ et al., 2019). Arjan de Groot and colleagues solved the crystal structure of DdrO from $D$. deserti and reported similar conclusions (de Groot et al., 2019). In a manner similar to the derepression of LexA, it is found by Laurence Blanchard that cleavage by PprI decreases the intracellular pool of unbound $\mathrm{DdrO}$, resulting in dissociation of RDRM-bound DdrO and leading to DDR gene transcription (Blanchard et al., 2017).

\section{HYPOTHETIC MECHANISMS OF PprI ACTIVATION}

In contrast to most genes related to the DNA damage response, the transcriptomic study by Liu et al. (2003) detected a constant level of pprI transcription during the early, middle, and late phases of recovery in $D$. radiodurans after acute irradiation at 15 $\mathrm{kGy}$, indicating an unclear activation mechanism of PprI. Several hypotheses have been proposed that might explain the activation of PprI since the activation mechanism of PprI after irradiation remained unknown (Figure 2A).

\section{The Release of $\mathrm{Zn}^{2+} / \mathrm{Mn}^{2+}$ Caused by Radiation or Oxidative Stress}

Blanchard discovered that the protease activity of PprI from $D$. deserti could be restored in the presence of $\mathrm{Zn}^{2+}, \mathrm{Mn}^{2+}$, or $\mathrm{Fe}^{2+}$ in vitro (Blanchard et al., 2017). As radiation and oxidative stress can result in the rapid release of $\mathrm{Zn}^{2+}$ from cysteinecontaining zinc sites, Qi suggested that the level of intracellular $\mathrm{Zn}^{2+}$ may be responsible for the activation of PprI (Maret, 2006; Kroncke and Klotz, 2009; Qi et al., 2020). Yet, it has to be demonstrated whether the level of intracellular $\mathrm{Zn}^{2+}$ increases. However, Wang reported that the protease activity of PprI from D. radiodurans depends on $\mathrm{Mn}^{2+}$ and that stimulation of PprI activity may rely on the alteration between $\mathrm{Mn}^{2+}$ and other ions (Wang et al., 2015).

\section{Posttranslational Modifications}

Posttranslational modification (PTM) has always been thought to be responsible for activating protein function in DNA damage response pathways, such as phosphorylation of H2A.X and ubiquitylation of $\mathrm{Ku}$ in DNA damage signaling and the NHEJ pathway, respectively (Kinner et al., 2008; Postow et al., 2008). Recently, Zhou et al. revealed the succinylome of $D$. radiodurans that is involved in its extreme resistance. In vitro assays have verified that glutamate substitution of Lys185 (K185E) in PprI, which mimics lysine succinylation, results in decreased enzymatic activity but that $\mathrm{K} 185 \mathrm{~A}$ exhibits enhanced protease activity (Zhou et al., 2019; Figure 2A). Whether other kinds of modification exist in PprI and affect the activation need further research.

\section{Small Molecules Binding to the GAF-Like Domain}

The GAF domains always bind small molecules such as cAMP and CGMP, which participate as secondary messengers in many cellular signal transduction pathways. The C-terminus of PprI exhibits a GAF-like domain, the function of which has not yet been demonstrated. Li proved that the addition of dGMP significantly enhanced $D$. radiodurans tolerance to $\mathrm{H}_{2} \mathrm{O}_{2}$ and gamma radiation by stimulating the activity of KatA and inducing the transcription of an extracellular nuclease (Dr_b0067) (Li et al., 2013). Based on the results, it is tempting to propose that PprI might be activated via a signaling molecule such as dGMP interacting with the GAF-like domain (Figure 2A). Regardless, the GAF domain of $D$. deserti PprI exhibits a "closed gate" (the long loop from residue 201 to residue 211 that connects the second and third strands), blocking the access to the hydrophobic pocket, which may function as a small molecule binding site (Vujicic-Zagar et al., 2009). Although this blocking may be caused by crystal stacking, unless the loop region is moved aside, otherwise, small molecules would be rejected into the pocket to activate PprI.

\section{POTENTIAL APPLICATIONS OF PprI IN GENETIC ENGINEERING}

It is meaningful to increase the resistance of organisms, especially industrial microbes and crops grown in extreme environments. In addition to mutant selection, overexpression of heterologous genes like the global regulator pprI from Deinococcus can help increase resistance to environmental stresses in organisms (Jin et al., 2019; Wang et al., 2019).

For example, Gao and Pan expressed Dr_pprI in Escherichia coli, which resulted in enhanced tolerances to radiation, salt, osmotic, reactive oxygen species (ROS) and other stresses. For example, the $\mathrm{D}_{10}$ dose of ionizing radiation increased from 50 to $250 \mathrm{~Gy}$, although it is quite far from that of Deinococcus (Gao et al., 2003; Pan et al., 2009). Ma also found that transduction of Dr_pprI into ethanologenic E. coli increases the ethanol production by 14.7 and $26.3 \%$ from glucose and xylose, respectively (Ma et al., 2011). Similarly, Dong produced a lactic 


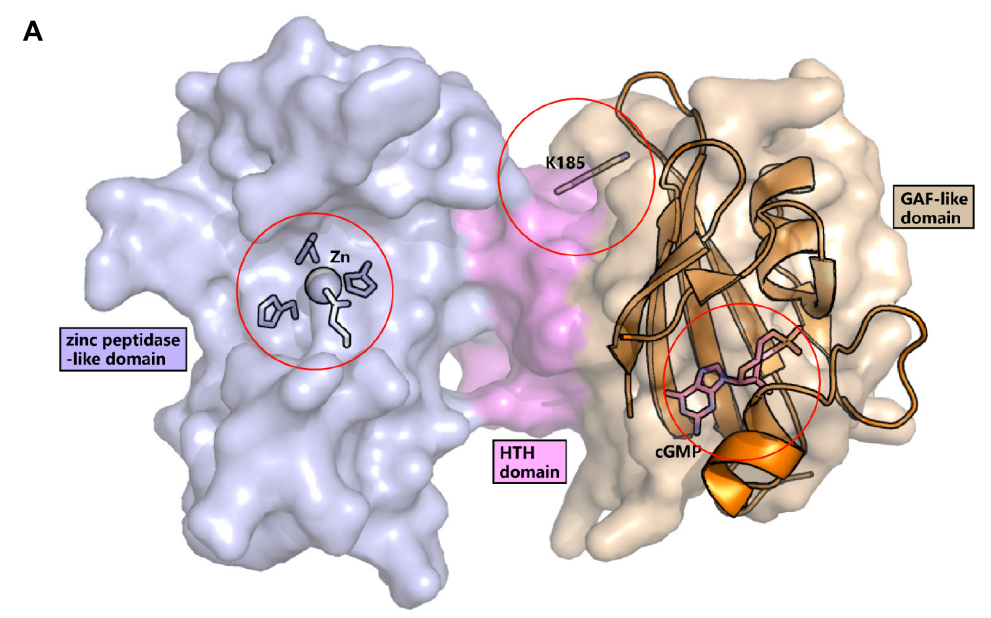

B

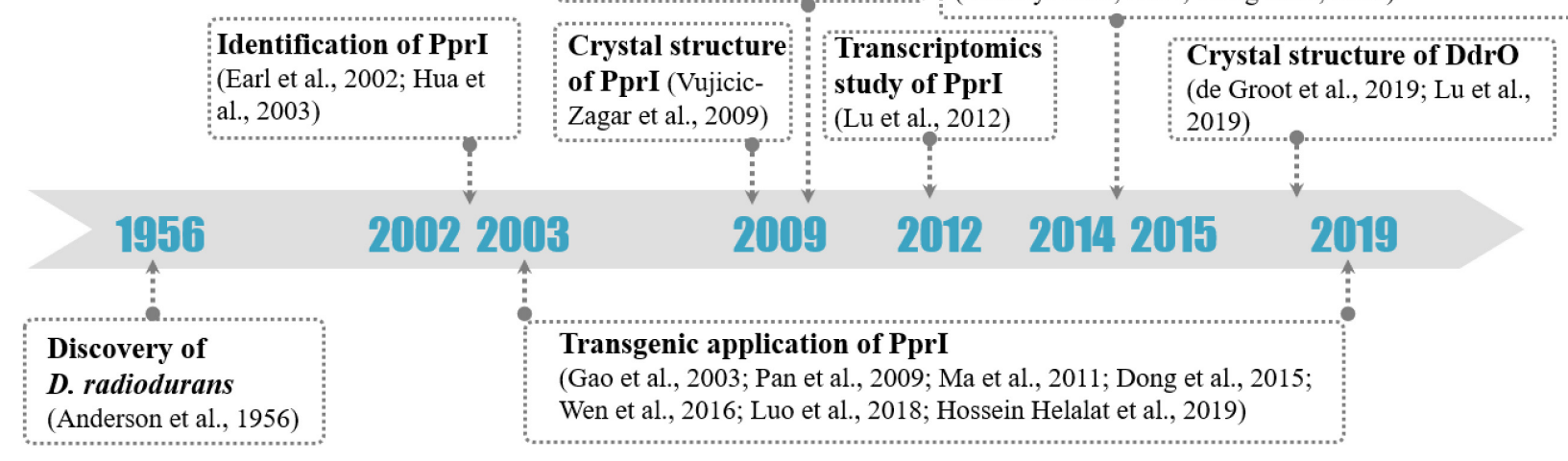

Identification and biochemical research of DdrO (Ludanyi et al., 2014; Wang et al., 2015)

FIGURE 2 | Activation hypotheses and timeline of important discoveries of Pprl. (A) Three hypotheses of the Pprl activation are present on the structure of DR_Pprl, which is from the homolog modeling (Swiss model) using D. deserti protein (PDB: 3DTE) as the starting model. DR_Pprl is shown as surface, and the zinc peptidase-like domain, helix-turn-helix motif and GAF-like domain are colored in light blue, magenta, and wheat, respectively. Related residues are shown as sticks. The aligned structure of PDE2A (RCSB PDB code 1MC0) is shown as cartoon. The four residues and metal ion in the NTD zinc peptidase-like domain represent the metal hypothesis. The Lys185 residue represents the posttranscriptional modification hypothesis. The cGMP in the GAF-like pocket by alignment represents the small molecule hypothesis. (B) Timeline of the important discoveries of Pprl.

acid high-yield and stress-tolerant strain of Lactococcus lactis by expression of pprI from $D$. radiodurans. The increment of lactic acid reached even up to threefold especially under salt stress (Dong et al., 2015). Luo explored the effects of introducing pprI into the electrochemically active bacterium Pseudomonas aeruginosa PAO1 and achieved an increase in power density by $71 \%$ higher than that of the control strain (Luo et al., 2018). The transduction of pprI works even in eukaryotes. Hossein Helalat reported that the heterologous expression of the pprI gene generated a 1.5 -fold alcohol and salt stress-tolerant strain of Saccharomyces cerevisiae (Hossein Helalat et al., 2019). Furthermore, Wen attempted to introduce pprI cloned in the pEGFP-c1 vector into mouse and human cells, and showed that its expression relieved acute radiation-induced damage to different organs and increased nearly $30 \%$ the survival rate by regulating expression of Rad51 (Wen et al., 2016). The detailed mechanism of how PprI affects the stress tolerance of other organisms remains unknown so far. One possible explanation is the similarity in analogous stress regulon systems. Adding PprI from Deinococcus may increase the amount of PprI-like protease copies and improve the survival in extreme environments to some point. The transcriptome and proteome in E. coli expressing PprI revealed the regulation of gene response not only to DNA damage but also to $\mathrm{pH}$ stress, and osmotic and oxidative stress, which also indicated the analogous stress regulon systems in E. coli (Zhou et al., 2011; Chen et al., 2012; Zhao et al., 2015). What is more, researchers have also inferred that PprI has other functions in addition to acting as a protease to derepress transcription in response to DNA damage. Further study will help to better reveal its functional mechanisms and can be applied to various human production activities.

\section{CONCLUSION}

Identified nearly 64 years ago, $D$. radiodurans has been designated as one of the most radio-resistant organisms on Earth (Anderson et al., 1956). The reason for its robust viability has been revealed 
with research progresses of the antioxidant system and DNA damage repair, especially when the essential of p prI for the stress resistance and its orchestrating on DNA damage genes such as $\operatorname{rec} A$ are confirmed (Earl et al., 2002; Hua et al., 2003; Longtin, 2003).

In the past 17 years, the pprI gene has been studied by genomics, transcriptomics, proteomics, bioinformatics, molecular biology, and structural biology approaches, revealing its structural and functional characteristics (Figure 2B). Structural data reveal the composition of three domains, suggesting its function as a protease, which was later demonstrated with the discovery of its specific substrate, DdrO. DNA microarrays and proteomics analysis have revealed that the pprI gene is responsible for regulating various genes participating in transcription, translation, metabolism, and DNA damage repair. However, transcriptomics data also suggest that the DNA damage response mediated by PprI does not rely on the induction of protein translation but on an unclear activation mechanism that needs further research.

Comparison of the PprI-DdrO response system with the SOS response system reveals distinctions between them. For one thing, the dimerization of the two repressors depends on the interaction from both NTD and CTD, while the interface of DdrO is much more extensive and CTD dependent (Lu et al., 2019; Chen et al., 2020). For another, the dissociation of LexA relies on the autocleavage, which is promoted by the stabilization of autocleavage conformation when RecA is activated after sensing DNA damage and form RecA-ssDNA-ATP filaments (Butala et al., 2008, 2011). On this occasion, the cleavage conducted by PprI is much more direct and efficient compared with the co-protease activity of RecA.

The efficiency of the PprI-DdrO response system also relies on the antioxidant intracellular environment protecting the proteome, which is provided and kept by the extraordinary antioxidant system (Daly et al., 2004, 2010; Slade and Radman, 2011). The domestication of the highresistant E. coli by 100-cycle selection exhibits reduced level of

\section{REFERENCES}

Anderson, A. W., Nordan, H. C., Cain, R. F., Parrish, G., and Duggan, D. (1956). Studies on a radio-resistant micrococcus .1. Isolation, morphology, cultural characteristics, and resistance to gamma radiation. Food Technol. 10, 575-578.

Aravind, L., and Ponting, C. P. (1997). The GAF domain: an evolutionary link between diverse phototransducing proteins. Trends Biochem. Sci. 22, 458-459. doi: 10.1016/s0968-0004(97)01148-1

Bauermeister, A., Bentchikou, E., Moeller, R., and Rettberg, P. (2009). Roles of PprA, IrrE, and RecA in the resistance of Deinococcus radiodurans to germicidal and environmentally relevant UV radiation. Arch. Microbiol. 191, 913-918. doi: 10.1007/s00203-009-0522-7

Blanchard, L., Guerin, P., Roche, D., Cruveiller, S., Pignol, D., Vallenet, D., et al. (2017). Conservation and diversity of the IrrE/DdrO-controlled radiation response in radiation-resistant Deinococcus bacteria. Microbiologyopen 6:e00477. doi: $10.1002 / \mathrm{mbo} 3.477$

Blasius, M., Sommer, S., and Hubscher, U. (2008). Deinococcus radiodurans: what belongs to the survival kit? Crit. Rev. Biochem. Mol. Biol. 43, 221-238. doi: 10.1080/10409230802122274

Bose, B., Auchtung, J. M., Lee, C. A., and Grossman, A. D. (2008). A conserved anti-repressor controls horizontal gene transfer by hydroxylation, which further indicates the relationship between DNA damage repair and antioxidant system (Bruckbauer et al., 2020). In other words, both DDR system and antioxidant system are important, without which the radiation resistance will be greatly impaired.

DNA damage occurs throughout the entire life cycle, inducing mutation, cancer and cell death, which are prevented by the DNA damage response that includes a series of activities such as DNA repair, cell cycle checkpoints, and apoptosis. Studies on the DNA damage response can contribute to the development of new drugs for cancer therapy, such as small molecule inhibitors that target key proteins in DNA damage response and repair pathways (Li et al., 2020). Furthermore, greater knowledge of DNA damage response mechanisms may help to prevent cancerinducing habits and guide healthy living. Research on the DNA damage response, such as the SOS and PprI-DdrO response systems, can help in elucidating the extraordinary resistance of Deinococcus and the mechanisms of organisms that can survive environmental stresses. Regardless, much work is needed to fully understand the multiple DNA damage response systems.

\section{AUTHOR CONTRIBUTIONS}

$\mathrm{HL}$ and YH reviewed the literature and wrote the manuscript. Both authors contributed to the article and approved the submitted version.

\section{FUNDING}

This work was supported by the National Key Research and Development Program of China (2017YFA0503900), the grants from National Natural Science Foundation of China (31670065 and 31870051), and China Postdoctoral Science Foundation (2020M671699).

proteolysis. Mol. Microbiol. 70, 570-582. doi: 10.1111/j.1365-2958.2008. 06414.x

Boulton, S. J., Gartner, A., Reboul, J., Vaglio, P., Dyson, N., Hill, D. E., et al. (2002). Combined functional genomic maps of the C. elegans DNA damage response. Science 295, 127-131. doi: 10.1126/science.106 5986

Bruckbauer, S. T., Martin, J., Minkoff, B. B., Veling, M. T., Lancaster, I., Liu, J., et al. (2020). Physiology of highly radioresistant Escherichia coli after experimental evolution for 100 cycles of selection. Front. Microbiol. 11:582590. doi: 10.3389/ fmicb. 2020.582590

Butala, M., Klose, D., Hodnik, V., Rems, A., Podlesek, Z., Klare, J. P., et al. (2011). Interconversion between bound and free conformations of LexA orchestrates the bacterial SOS response. Nucleic Acids Res. 39, 6546-6557. doi: 10.1093/nar/ gkr265

Butala, M., Žgur-Bertok, D., and Busby, S. J. W. (2008). The bacterial LexA transcriptional repressor. Cellular and Molecular Life Sciences 66, 82-93. doi: 10.1007/s00018-008-8378-6

Chen, H., Xu, G., Zhao, Y., Tian, B., Lu, H., Yu, X., et al. (2008). A novel OxyR sensor and regulator of hydrogen peroxide stress with one cysteine residue in Deinococcus radiodurans. PLoS One 3:e1602. doi: 10.1371/journal. pone.0001602 
Chen, T., Wang, J., Zeng, L., Li, R., Li, J., Chen, Y., et al. (2012). Significant rewiring of the transcriptome and proteome of an Escherichia coli strain harboring a tailored exogenous global regulator IrrE. PLoS One 7:e37126. doi: 10.1371/ journal.pone.0037126

Chen, Z., Tang, Y., Hua, Y., and Zhao, Y. (2020). Structural features and functional implications of proteins enabling the robustness of Deinococcus radiodurans. Comput. Struct. Biotechnol. J. 18, 2810-2817. doi: 10.1016/j.csbj.2020.09.036

Cox, M. M., and Battista, J. R. (2005). Deinococcus radiodurans - the consummate survivor. Nat. Rev. Microbiol. 3, 882-892. doi: 10.1038/nrmicro1264

Daly, M. J., Gaidamakova, E. K., Matrosova, V. Y., Kiang, J. G., Fukumoto, R., Lee, D. Y., et al. (2010). Small-molecule antioxidant proteome-shields in Deinococcus radiodurans. PLoS One 5:e12570. doi: 10.1371/journal.pone. 0012570

Daly, M. J., Gaidamakova, E. K., Matrosova, V. Y., Vasilenko, A., Zhai, M., Venkateswaran, A., et al. (2004). Accumulation of $\mathrm{Mn}$ (II) in Deinococcus radiodurans facilitates gamma-radiation resistance. Science 306, 1025-1028. doi: 10.1126/science.1103185

de Groot, A., Siponen, M. I., Magerand, R., Eugenie, N., Martin-Arevalillo, R., Doloy, J., et al. (2019). Crystal structure of the transcriptional repressor DdrO: insight into the metalloprotease/repressor-controlled radiation response in Deinococcus. Nucleic Acids Res. 47, 11403-11417. doi: 10.1093/nar/ gkz883

Devigne, A., Ithurbide, S., Bouthier de la Tour, C., Passot, F., Mathieu, M., Sommer, S., et al. (2015). DdrO is an essential protein that regulates the radiation desiccation response and the apoptotic-like cell death in the radioresistant Deinococcus radiodurans bacterium. Mol. Microbiol. 96, 1069-1084. doi: 10. 1111/mmi.12991

Dong, X., Tian, B., Dai, S., Li, T., Guo, L., Tan, Z., et al. (2015). Expression of PprI from Deinococcus radiodurans improves lactic acid production and stress tolerance in Lactococcus lactis. PLoS One 10:e0142918. doi: 10.1371/journal. pone. 0142918

Earl, A. M., Mohundro, M. M., Mian, I. S., and Battista, J. R. (2002). The IrrE protein of Deinococcus radiodurans $\mathrm{R} 1$ is a novel regulator of recA expression. J. Bacteriol. 184, 6216-6224. doi: 10.1128/jb.184.22.6216-6224.2002

Gao, G., Tian, B., Liu, L., Sheng, D., Shen, B., and Hua, Y. (2003). Expression of Deinococcus radiodurans PprI enhances the radioresistance of Escherichia coli. DNA Repair. 2, 1419-1427. doi: 10.1016/j.dnarep.2003.08.012

Gao, G. J., Lu, H. M., Huang, L. F., and Hua, Y. J. (2005). Construction of DNA damage response gene pprI function-deficient and function-complementary mutants in Deinococcus radiodurans. Chin. Sci. Bull. 50, 311-316. doi: 10.1360/ 982004-719

Hossein Helalat, S., Bidaj, S., Samani, S., and Moradi, M. (2019). Producing alcohol and salt stress tolerant strain of Saccharomyces cerevisiae by heterologous expression of pprI gene. Enzyme Microb. Technol. 124, 17-22. doi: 10.1016/j. enzmictec.2019.01.008

Hua, Y., Narumi, I., Gao, G., Tian, B., Satoh, K., Kitayama, S., et al. (2003). PprI: a general switch responsible for extreme radioresistance of Deinococcus radiodurans. Biochem. Biophys. Res. Commun. 306, 354-360. doi: 10.1016/ s0006-291x(03)00965-3

Jin, M., Xiao, A., Zhu, L., Zhang, Z., Huang, H., and Jiang, L. (2019). The diversity and commonalities of the radiation-resistance mechanisms of Deinococcus and its up-to-date applications. AMB Express 9:138. doi: 10.1186/s13568-0190862-x

Jolivet, E., Lecointe, F., Coste, G., Satoh, K., Narumi, I., Bailone, A., et al. (2006). Limited concentration of RecA delays DNA double-strand break repair in Deinococcus radiodurans R1. Mol. Microbiol. 59, 338-349. doi: 10.1111/j.13652958.2005.04946.x

Kinner, A., Wu, W., Staudt, C., and Iliakis, G. (2008). Gamma-H2AX in recognition and signaling of DNA double-strand breaks in the context of chromatin. Nucleic Acids Res. 36, 5678-5694. doi: 10.1093/nar/gkn550

Kota, S., Charaka, V. K., Ringgaard, S., Waldor, M. K., and Misra, H. S. (2014). PprA contributes to Deinococcus radiodurans resistance to nalidixic acid, genome maintenance after DNA damage and interacts with deinococcal topoisomerases. PLoS One 9:e85288. doi: 10.1371/journal.pone.0085288

Kroncke, K. D., and Klotz, L. O. (2009). Zinc fingers as biologic redox switches? Antioxid Redox Signal. 11, 1015-1027. doi: 10.1089/ARS.2008.2269

Li, M., Sun, H., Feng, Q., Lu, H., Zhao, Y., Zhang, H., et al. (2013). Extracellular dGMP enhances Deinococcus radiodurans tolerance to oxidative stress. PLoS One 8:e54420. doi: 10.1371/journal.pone. 0054420

Li, L., Kumar, A. K., Hu, Z., and Guo, Z. (2020). Small molecule inhibitors targeting the key proteins in DNA damage response for cancer therapy. Curr. Med.Chem. 27, 1-21. doi: 10.2174/0929867327666200224102309

Lim, S., Jung, J. H., Blanchard, L., and de Groot, A. (2019). Conservation and diversity of radiation and oxidative stress resistance mechanisms in Deinococcus species. FEMS Microbiol. Rev. 43, 19-52. doi: 10.1093/femsre/fuy037

Liu, Y., Zhou, J., Omelchenko, M. V., Beliaev, A. S., Venkateswaran, A., Stair, J., et al. (2003). Transcriptome dynamics of Deinococcus radiodurans recovering from ionizing radiation. Proc. Natl. Acad. Sci. U.S.A. 100, 4191-4196. doi: $10.1073 /$ pnas. 0630387100

Longtin, R. (2003). Deinoccocus radiodurans: getting a better fix on DNA repair. J. Natl. Cancer Inst. 95, 1270-1271. doi: 10.1093/jnci/95.17.1270

Lu, H., Chen, H., Xu, G., Shah, A. M., and Hua, Y. (2012). DNA binding is essential for PprI function in response to radiation damage in Deinococcus radiodurans. DNA Repair. 11, 139-145. doi: 10.1016/j.dnarep.2011.10.013

Lu, H., Gao, G., Xu, G., Fan, L., Yin, L., Shen, B., et al. (2009). Deinococcus radiodurans PprI switches on DNA damage response and cellular survival networks after radiation damage. Mol. Cell Proteomics 8, 481-494. doi: 10.1074/ mcp.M800123-MCP200

Lu, H., Wang, L., Li, S., Pan, C., Cheng, K., Luo, Y., et al. (2019). Structure and DNA damage-dependent derepression mechanism for the XRE family member DG-DdrO. Nucleic Acids Res. 47, 9925-9933. doi: 10.1093/nar/gkz720

Ludanyi, M., Blanchard, L., Dulermo, R., Brandelet, G., Bellanger, L., Pignol, D., et al. (2014). Radiation response in Deinococcus deserti: IrrE is a metalloprotease that cleaves repressor protein DdrO. Mol. Microbiol. 94, 434-449. doi: 10.1111/ mmi. 12774

Luo, J., Wang, T., Li, X., Yang, Y., Zhou, M., Li, M., et al. (2018). Enhancement of bioelectricity generation via heterologous expression of IrrE in Pseudomonas aeruginosa-inoculated MFCs. Biosens. Bioelectron. 117, 23-31. doi: 10.1016/j. bios.2018.05.052

Ma, R., Zhang, Y., Hong, H., Lu, W., Lin, M., Chen, M., et al. (2011). Improved osmotic tolerance and ethanol production of ethanologenic Escherichia coli by IrrE, a global regulator of radiation-resistance of Deinococcus radiodurans. Curr. Microbiol. 62, 659-664. doi: 10.1007/s00284-010-9759-2

Makarova, K. S., Omelchenko, M. V., Gaidamakova, E. K., Matrosova, V. Y., Vasilenko, A., Zhai, M., et al. (2007). Deinococcus geothermalis: the pool of extreme radiation resistance genes shrinks. PLoS One 2:e955. doi: 10.1371/ journal.pone.0000955

Makarova, K. S., Wolf, Y. I., and Koonin, E. V. (2009). Comprehensive comparative-genomic analysis of type 2 toxin-antitoxin systems and related mobile stress response systems in prokaryotes. Biol. Direct, 4:19. doi: 10.1186/ 1745-6150-4-19

Maret, W. (2006). Zinc coordination environments in proteins as redox sensors and signal transducers. Antioxid Redox Signal. 8, 1419-1441. doi: 10.1089/ars. 2006.8.1419

Martinez, S. E., Wu, A. Y., Glavas, N. A., Tang, X. B., Turley, S., Hol, W. G., et al. (2002). The two GAF domains in phosphodiesterase 2A have distinct roles in dimerization and in cGMP binding. Proc. Natl. Acad. Sci. U.S.A, 99, 13260-13265. doi: 10.1073/pnas.192374899

Narumi, I., Satoh, K., Kikuchi, M., Funayama, T., Yanagisawa, T., Kobayashi, Y., et al. (2001). The LexA protein from Deinococcus radiodurans is not involved in RecA induction following gamma irradiation. J. Bacteriol. 183, 6951-6956. doi: 10.1128/JB.183.23.6951-6956.2001

Pan, J., Wang, J., Zhou, Z., Yan, Y., Zhang, W., Lu, W., et al. (2009). IrrE, a global regulator of extreme radiation resistance in Deinococcus radiodurans, enhances salt tolerance in Escherichia coli and Brassica napus. PLoS One 4:e4422. doi: 10.1371/journal.pone.0004422

Pilzecker, B., Buoninfante, O. A., and Jacobs, H. (2019). DNA damage tolerance in stem cells, ageing, mutagenesis, disease and cancer therapy. Nucleic Acids Res. 47, 7163-7181. doi: 10.1093/nar/gkz531

Postow, L., Ghenoiu, C., Woo, E. M., Krutchinsky, A. N., Chait, B. T., and Funabiki, H. (2008). Ku80 removal from DNA through double strand break-induced ubiquitylation. J. Cell Biol. 182, 467-479. doi: 10.1083/jcb.200802146

Qi, H. Z., Wang, W. Z., He, J. Y., Ma, Y., Xiao, F. Z., and He, S. Y. (2020). Antioxidative system of Deinococcus radiodurans. Res. Microbiol. 171, 45-54. doi: 10.1016/j.resmic.2019.11.002 
Radman, M. (1975). SOS repair hypothesis: phenomenology of an inducible DNA repair which is accompanied by mutagenesis. Basic Life Sci. 5A, 355-367. doi: 10.1007/978-1-4684-2895-7_48

Selvam, K., Duncan, J. R., Tanaka, M., and Battista, J. R. (2013). DdrA, DdrD, and PprA: components of UV and mitomycin C resistance in Deinococcus radiodurans $\mathrm{R} 1$. PLoS One 8:e69007. doi: 10.1371/journal.pone.0069007

Sevvana, M., Vijayan, V., Zweckstetter, M., Reinelt, S., Madden, D. R., HerbstIrmer, R., et al. (2008). A ligand-induced switch in the periplasmic domain of sensor histidine kinase CitA. J. Mol. Biol. 377, 512-523. doi: 10.1016/j.jmb.2008. 01.024

Sharma, A., Gaidamakova, E. K., Grichenko, O., Matrosova, V. Y., Hoeke, V., Klimenkova, P., et al. (2017). Across the tree of life, radiation resistance is governed by antioxidant $\mathrm{Mn}(2+)$, gauged by paramagnetic resonance. Proc. Natl. Acad. Sci. U.S.A. 114, E9253-E9260. doi: 10.1073/pnas.171360 8114

Sheng, D., Zheng, Z., Tian, B., Shen, B., and Hua, Y. (2004). LexA analog (dra0074) is a regulatory protein that is irrelevant to recA induction. J. Biochem. 136, 787-793. doi: 10.1093/jb/mvh188

Singh, H., Appukuttan, D., and Lim, S. (2014). Hsp20, a small heat shock protein of Deinococcus radiodurans, confers tolerance to hydrogen peroxide in Escherichia coli. J. Microbiol. Biotechnol. 24, 1118-1122. doi: 10.4014/jmb.1403. 03006

Slade, D., and Radman, M. (2011). Oxidative stress resistance in Deinococcus radiodurans. Microbiol. Mol. Biol. Rev. 75, 133-191. doi: 10.1128/MMBR. 00015-10

Vujicic-Zagar, A., Dulermo, R., Le Gorrec, M., Vannier, F., Servant, P., Sommer, S., et al. (2009). Crystal structure of the IrrE protein, a central regulator of DNA damage repair in deinococcaceae. J. Mol. Biol. 386, 704-716. doi: 10.1016/j.jmb. 2008.12.062

Wang, L., Xu, G., Chen, H., Zhao, Y., Xu, N., Tian, B., et al. (2008). DrRRA: a novel response regulator essential for the extreme radioresistance of Deinococcus radiodurans. Mol. Microbiol. 67, 1211-1222. doi: 10.1111/j.1365-2958.2008. 06113.x

Wang, L. Y., Yin, L. F., Xu, G. Z., Li, M. F., Zhang, H., Tian, B., et al. (2012). Cooperation of PprI and DrRRA in response to extreme ionizing radiation in Deinococcus radiodurans. Chin. Sci. Bull. 57, 98-104. doi: 10.1007/s11434-0114790-7
Wang, W., Ma, Y., He, J., Qi, H., Xiao, F., and He, S. (2019). Gene regulation for the extreme resistance to ionizing radiation of Deinococcus radiodurans. Gene 715:144008. doi: 10.1016/j.gene.2019.144008

Wang, Y., Xu, Q., Lu, H., Lin, L., Wang, L., Xu, H., et al. (2015). Protease activity of PprI facilitates DNA damage response: Mn2+-dependence and substrate sequence-specificity of the proteolytic reaction. PLoS One 10:e0122071. doi: 10.1371/journal.pone.0122071

Wen, L., Yue, L., Shi, Y., Ren, L., Chen, T., Li, N., et al. (2016). Deinococcus radiodurans pprI expression enhances the radioresistance of eukaryotes. Oncotarget 7, 15339-15355. doi: 10.18632/oncotarget.8137

Yang, S., Xu, H., Wang, J., Liu, C., Lu, H., Liu, M., et al. (2016). Cyclic AMP Receptor Protein Acts as a Transcription Regulator in Response to Stresses in Deinococcus radiodurans. PLoS One 11:e0155010. doi: 10.1371/journal.pone. 0155010

Zhao, P., Zhou, Z., Zhang, W., Lin, M., Chen, M., and Wei, G. (2015). Global transcriptional analysis of Escherichia coli expressing IrrE, a regulator from Deinococcus radiodurans, in response to $\mathrm{NaCl}$ shock. Mol. Biosyst. 11, 11651171. doi: 10.1039/c5mb00080g

Zhou, C., Dai, J., Lu, H., Chen, Z., Guo, M., He, Y., et al. (2019). Succinylome analysis reveals the involvement of lysine succinylation in the extreme resistance of Deinococcus radiodurans. Proteomics 19:e1900158. doi: 10.1002/ pmic. 201900158

Zhou, Z., Zhang, W., Chen, M., Pan, J., Lu, W., Ping, S., et al. (2011). Genomewide transcriptome and proteome analysis of Escherichia coli expressing IrrE, a global regulator of Deinococcus radiodurans. Mol. Biosyst. 7, 1613-1620. doi: $10.1039 / \mathrm{c} 0 \mathrm{mb} 00336 \mathrm{k}$

Conflict of Interest: The authors declare that the research was conducted in the absence of any commercial or financial relationships that could be construed as a potential conflict of interest.

Copyright (c) $2021 \mathrm{Lu}$ and Hua. This is an open-access article distributed under the terms of the Creative Commons Attribution License (CC BY). The use, distribution or reproduction in other forums is permitted, provided the original author(s) and the copyright owner(s) are credited and that the original publication in this journal is cited, in accordance with accepted academic practice. No use, distribution or reproduction is permitted which does not comply with these terms. 